\title{
Effect of UBE2Q on BECN and CEA Protein in Colorectal Cancer Cells
}

\section{Niloofar Boroumand}

Department of Biotechnology, Faculty of Advanced Sciences and Technologies, University of Isfahan, Isfahan, Iran

\section{Abstract}

Background: Expression of UBE2Q1, UBE2Q2, and members of the ubiquitinconjugating enzyme family (E2) are affected in colorectal cancer (CRC). The BECN gene plays a key role in CRC cells. In gastrointestinal carcinoma therapy, tumorassociated antigens such as CEA are typically used.To investigate the association between UBE2Q1 and Beclin1 autophagy marker and CEA protein expression in LS180 CRC cell line.

Materials and Methods: In this study, changes in the expression of BECN marker in LS180 cell lines with the vector containing UBE2Q1 were investigated using real-time PCR. The expression of CEA protein was also evaluated by western blotting. Statistical analyses were performed with Graph Pad Prism software.

Results: The results indicated reduced expression of $B E C N$ autophagy marker $(\mathrm{P}=0)$. Therefore, in the presence of UBE2Q1, cancer cells have less ability to induce autophagy. However, CEA protein levels in LS180 transfected cells with a UBE2Q1-

Corresponding Author: Niloofar Boroumand; email: Nilufar-

boroumand@gmail.com

Production and Hosting by

Knowledge E

(c) Niloofar Boroumand. This article is distributed under the terms of the Creative

Commons Attribution

License, which permits unrestricted use and redistribution provided that the original author and source are credited. Editor-in-Chief: Dr. Alireza Rafiei

\section{G OPEN ACCESS} ORF-containing plasmid decreased when compared to non-transfected cells.

Conclusion: The use of pharmacologic inhibitors related to the autophagy mechanism can be a novel approach in cancer therapy.

Keywords: BECN, CEA protein, colorectal cancer

\section{Introduction}

Colorectal cancer (CRC) is the third leading cause of cancer death among both men and women. In this disease malignant cells form in colon tissues [1]. It is recommended that women and men aged 50 years and older should have either a fecal occult blood test (FOBT) annually, a sigmoidoscopy or double contrast barium enema (DCBE) every 5 years, or a colonoscopy every 10 years [2]. The risk of developing CRC due to obesity is estimated to be $20 \%$, with an increased risk of malignancies being influenced by diet and weight change [3]. Studies have proposed that a history of CRC in a first-degree relative (a parent or sibling) raises one's lifetime risk of CRC 1.8-fold to 8.0-fold [4]. Research on the creation and control mechanisms of cancer has shown that some genes are affected during the progression of CRC [5]. Autophagy is a proteolytic self-destruction mechanism and a cytoplasmic disintegration agent within cells that plays a role in the suppression and progression of tumor activity [6]. Autophagy 
has a fundamental role in cancer, and perturbations in autophagy can contribute to malignant disease [7]. Changes in autophagy impact some diseases such as CRC [8]. The ubiquitin-proteasome system has been shown to be activated in CRC and other malignancies [9]. UBE2Q1 and UBE2Q2 are well known cancer-related ubiquitinconjugating enzymes and are also known as E2 enzymes. These enzymes have a critical role in the regulated degradation of cellular proteins. An increase in the expression of $U B E 2 Q 1$ and $U B E 2 Q 2$ genes has been reported in cancer tissues and colorectal cancer cell lines [10]. BECN1 is the orthologous mammalian protein of yeast ATG6, which codes for the beclin-1 protein [11]. BECN1 plays an opposite role in CRC by protecting the tumor, but it may inhibit the growth of CRC cells. An increased expression of BECN1 in CRC colon tissue has been reported when compared to normal colorectal mucosa [12-13]. Carcinoembryonic antigen (CEA) is a glycoprotein in the cell membrane of many tissues, including cancerous colon and rectum. CEA is one of the most common tumor markers. This antigen is upregulated in diseases such as CRC and breast, lung, prostate, and stomach cancer [14-15]. Moreover, CEA proteins increase in CRC and can to devise therapy or study resistance to the therapy against CRC [16]. Therefore, the aim of this study was to investigate the relationship expression between UBE2Q1 expression and the BECN1 marker as well as CRC prognosis and increased expression of UBE2Q1 gene through the CEA protein.

\section{Materials and Methods}

\subsection{RNA and protein extraction}

RNA and protein were extracted using TRizol reagent (Invitrogen, USA) based on the manufacturer's instruction. The CRC cell line, LS180, was divided into two groups: one transfected with the HumanUBE2Q1cDNA (nm-017582.6) clone of pCMV6-AN-GFP and one transfected with the pCMV6-AN-GFP vector and lacking UBE2Q1. ORF was obtained from a previous study [17]. Quality of RNA was determined at a 260/280 nm wavelength ratio measured by a NanoDrop spectrometer (Thermo Scientific, Waltham, MA, USA).

\section{2. cDNA synthesis and real-time PCR}

cDNA synthesis was performed using a commercial kit (Fermentase, Lithuania), according to the manufacturer's instructions. Real-time PCR was performed in triplicate using an ABI PRISM 7500 instrument (Applied Biosystems, USA) in a total volume of $20 \mu \mathrm{L}$. Briefly, $5 \mu \mathrm{L}$ of cDNA produced was added to a master mix, including $1 \mathrm{pmol} \mu \mathrm{L}^{-1}$ of BECN and RPLPO (as a housekeeping gene) primers, $10 \mu \mathrm{l}$ of SYBR premix ExTaq II (TaKaRa, Kusatsu, Shiga Prefecture, Japan) and $3 \mu \mathrm{L}$ DEPC water (Sina gene, Iran). Primers were designed by the National Center for Biotechnology Information (NCBI) website and gene runner software. The uniqueness of the primers was confirmed by the BLAST program (Table 1). The run program was set at $72^{\circ} \mathrm{C}$ for $15 \mathrm{sec}$ followed by 40 cycles of $58^{\circ} \mathrm{C}$ for $15 \mathrm{sec}, 94^{\circ} \mathrm{C}$ for $30 \mathrm{sec}$, and $94^{\circ} \mathrm{C}$ for $5 \mathrm{~min}$. The efficiency of the primer was investigated at 5 concentrations (Figure 1). 
TABLE 1: Sequences of the primers.

\begin{tabular}{l|l|l|}
\hline Gene & Forward Primer & Reverse Primer \\
\hline BECN & GTTTGAGATGCACAGTTG & CAAACTCTACGTGTCAAC \\
\hline RPLPO & GTTGTGTGAGTGGAGAGG & CAACACACTCACCTCTCC \\
\hline
\end{tabular}

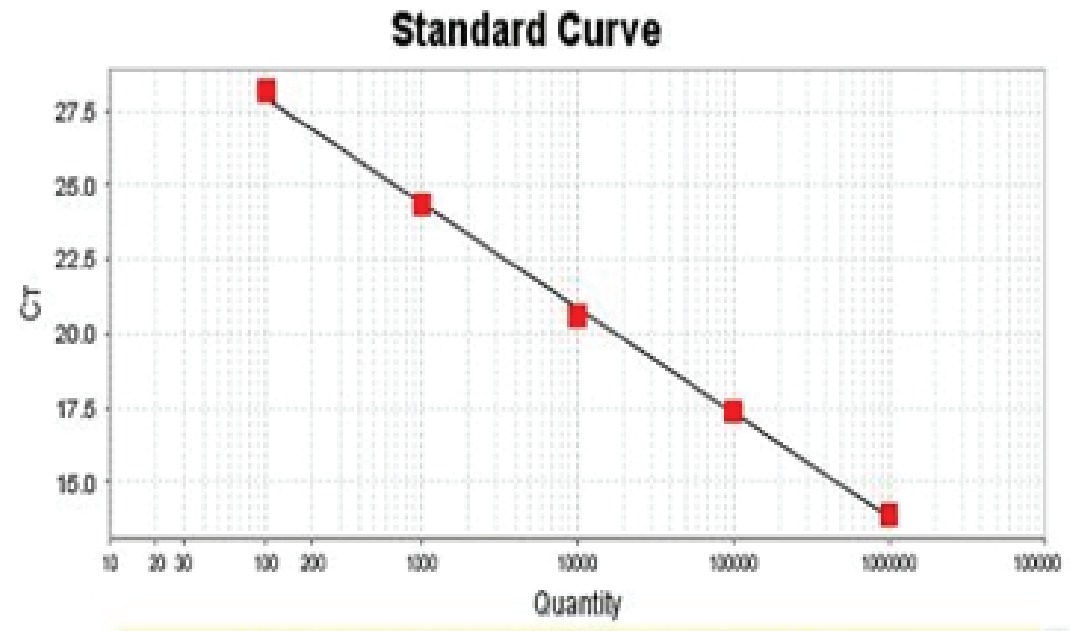

Taraet: BECN1 Slove: -3.564 Y.thter: $35.191 \mathrm{a}^{2}, 0.997$ Err\%: 90.816

Figure 1: Standard curve for different dilutions of real-time PCR products of the BECN gene.

\subsection{Western blot analysis}

Western blotting was performed using CEA antibody (1:1000 dilution; Can Ag, Sweden), rabbit anti-HRP enzyme (1:2000 dilution; Can Ag, Sweden), and ß-actin monoclonal antibody (1:1000 dilution; Abcam, USA), which were then reacted with the corresponding secondary HRP-conjugated antibodies (1:3000 dilution; Abcam, USA). Finally, blots were developed using the ECL Western Blotting Detection system (Amersham).

\subsection{Statistical analysis}

Real-time PCR data were analyzed using the $\triangle \triangle C T$ method, and final data were normalized by RPLPO, as an endogenous. Graph Pad Prism statistical software version 5.01 (Graph Pad, San Diego, CA, USA) was used for statistical analysis. The normality was evaluated by the Kolmogorov-Smirnov test. Mann-Whitney test was used to compare the groups. For all tests, a P-value of $<0.05$ was considered statistically significant. 


\section{Results}

\subsection{BECN expression after vector transfection with UBE2Q1 gene and empty vector}

The relative expression of the BECN gene in the LS180 cells was investigated 24 hours after transfection. BECN expression in UBE2Q1-ORF (44/51 $\pm 9 / 28)$ vector-transfected cells was found to be significantly reduced compared with that in the empty vector $(1 \pm 0)$ transfected cells $(\mathrm{P}=0)$ (Figure 2). Furthermore, results indicated that $B E C N$ expression in UBE2Q1-ORF (72/26 $\pm 29 / 08)$ vector-transfected cells was significantly reduced compared with that in the empty vector $(1 \pm 0)$ transfected cells $(P=0)$ (Figure 3$)$.

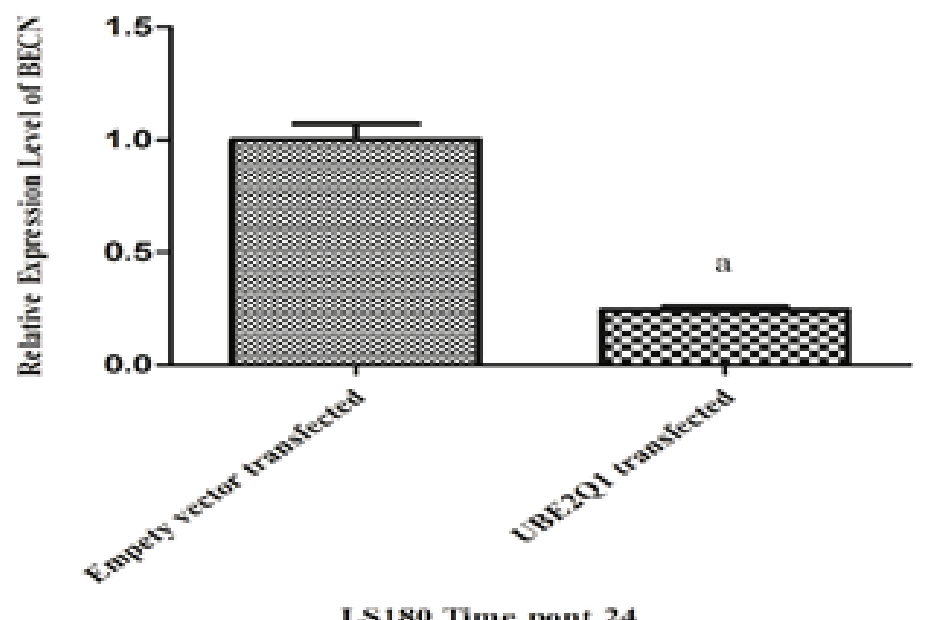

Figure 2: Relative expression of BECN gene in UBE2Q1-ORF vector-transfected cells and the empty vector transfected cells after 24 hours. Results are normalized to those of controls and are represented relative to expression of the RPLPO gene. (a) represents a significant difference between the groups $(\mathrm{P}=0)$. Error bars show mean $\pm \mathrm{SD}$.

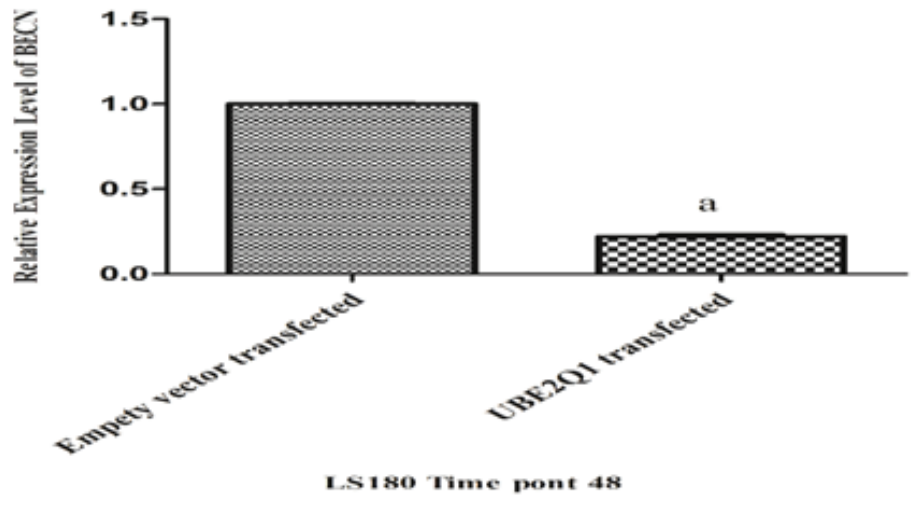

Figure 3: Relative expression of the BECN gene in UBE2Q1-ORF vector-transfected cells and the empty vector transfected cells after 48 hours. Results are normalized to those of controls and are represented relative to expression of the RPLPO gene. (a) represents significant differences between the groups $(P=0)$. Error bars show the mean \pm SD. 


\subsection{CEA protein expression}

Western blot analysis demonstrated that CEA protein levels in the LS180 cells transfected with a UBE2Q1-ORF containing plasmid 24 (Figure 4-A) and 48 (Figure 4-B) hours after transfection decreased compared to cells transfected with empty vectors and non-transfected cells.

A

2

1

3

A:

\section{3}

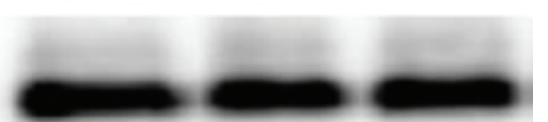

B

1

3

2

CEA

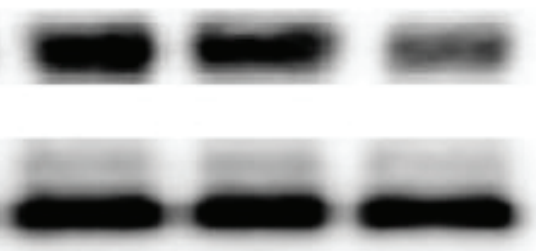

B: $\beta$ actin

Figure 4: CEA protein expression in the LS180 cell line 24 hours (A) and 48 hours (B) after transfection. 1: Non-transfected cells. 2: Transfected cells with empty vector. 3: Transfected cells with the UBE2Q1-ORF vector. $\beta$-actin antibody was used to confirm normalization of the protein load.

\section{Discussion}

In this study, we investigated BECN expression in LS180 cells transfected with UBE2Q using real-time PCR. Moreover, the expression of CEA protein was evaluated by western blot analysis. The results indicated a significant reduction in $B E C N$ gene expression in UBE2Q1 vector transfected cells compared to the group containing an empty vector. Western blot results showed a decrease in CEA protein levels in the LS180 cell line compared to the control. CEA protein levels can be used as an indicator for evaluating response to treatment or resistance to treatment. Therefore, reduction in CEA expression in LS180 cells can be an important factor in the selection and analysis of therapeutic methods for CRC. Recently, studies on cancer control have shown that some genes are involved in cancer progression [18]. Autophagy may help reduce metastasis in the early stages of tumor cell release [19]. Shafie et al (2013) examined the model of expression of the UBE2Q2 gene in eight CRC cell lines. The SW742 cell line showed the highest expression of UBE2Q2 at both levels of $\mathrm{mRNA}$ and protein with a significant increase in expression in 65.11\% CRC cases [9]. Chang et al (2015) investigated UBE2Q1 expression patterns in hepatocellular carcinoma cell lines and tissue samples. Also, western blot results showed increased UBE2Q1 expression in tumor tissues compared to normal cells [20]. The BECN1 gene was identified as the first autophagy marker in human CRC [21]. Zuli Yang et al (2015) studied Beclin-1 levels in CRC cells and found that Beclin-1 expression was higher in CRC cells than in normal cells [22]. Reduction of expression of autophagy marker (BECN) in the LS180 cell line suggests that in the presence of UBE2Q1, cancer cells have reduced ability to induce autophagy. Reducing the BECN expression in LS180 cells may increase ROS, which causes cellular damage to cancer cells through 
oxidative damage. Thus, reduction in autophagy and the increase in ROS may cause the cancer cell susceptibility. CEA is one of the most common tumor markers and is found in the cell membrane of many tissues, including colon cancer tissue and rectum [23]. Measuring the level of CEA is a suitable indicator for relapse after surgical removal of the tumor and for evaluating response or resistance to treatment [24]. Reduction in CEA expression in the UBE2Q1 vector-transfected group compared to the non-transfected control group and the empty vector transfected group could be a sign of improvement in prognosis in this group. However, more experimental loss and gain of function studies are needed to confirm this hypothesis.

\section{Conclusion}

The results of this study indicate that a reduction of CEA and BECN expression can be an important factor in the selection of therapeutic methods for CRC in the future. Reducing the expression level of these tumor markers in LS180 cells with the vector containing UBE2Q1 can be applied for the selection and analysis of therapeutic methods for CRC.

\section{Acknowledgments}

I thank all persons who contributed to this study.

\section{Conflicts of Interest}

The author declares no conflict of interest.

\section{References}

[1] Friedemann-Sánchez G, Griffin JM, Partin MR. Gender differences in colorectal cancer screening barriers and information needs 1. Health Expect. 2007;10(2):148-60.

[2] Smith RA, Cokkinides V, Eyre HJ. American Cancer Society guidelines for the early detection of cancer, 2003. CA Cancer J Clin. 2003;53(1):27-43.

[3] De Pergola G, Silvestris F. Obesity as a major risk factor for cancer. J Obes. 2013;2013.

[4] Fuchs CS, Giovannucci EL, Colditz GA, Hunter DJ, Speizer FE, Willett WC. A prospective study of family history and the risk of colorectal cancer. N Engl J Med. 1994;331(25):1669-74.

[5] Garraway LA, Lander ES. Lessons from the cancer genome. Cell. 2013; 153(1):17-37.

[6] Loos B, Engelbrecht AM, Lockshin RA, Klionsky DJ, Zakeri Z. The variability of autophagy and cell death susceptibility: Unanswered questions. Autophagy. 2013:9(9):1270-85.

[7] Jiang $X$, Overholtzer M, Thompson CB. Autophagy in cellular metabolism and cancer. J Clin Invest. 2015; 125:47-54.

[8] Kenific CM, Debnath J. Cellular and metabolic functions for autophagy in cancer cells. Trends Cell Biol. 2015; 25(1): 37-45

[9] Shafiee SM, Seghatoleslam A, Nikseresht M, Hosseini SV, Alizadeh-Naeeni M, Safaei A, Owji AA. UBE2Q1 expression in human colorectal tumors and cell lines. Mol Biol Rep. 2013; 40(12): 7045-51.

[10] Chang R, Wei L, Lu Y, Cui X, Lu C, Liu L. Upregulated expression of ubiquitin-conjugating enzyme E2Q1 (UBE2Q1) is associated with enhanced cell proliferation and poor prognosis in human hapatocellular carcinoma. J Mol Histol. 2015; 46(1): 45-56.

[11] Brooks CL, Gu W. Ubiquitination, phosphorylation and acetylation: the molecular basis for p53 regulation. Curr Opin Cell Biol. 2003; 15(2): 164-71. 
[12] Kang R, Zeh HJ, Lotze MT, Tang D. The Beclin 1 network regulates autophagy and apoptosis. Cell Death Differ. 2011; 18(4): 571-80.

[13] Yu L, McPhee CK, Zheng L, Mardones GA, Rong Y, Peng J. Termination of autophagy and reformation of lysosomes regulated by mTOR. Nature. 2010; 465(7300):942-6.

[14] Pihl E, Nairn RC, Hughes ES, Cuthbertson AM, Rollo AJ. Mucinous colorectal carcinoma: immunopathology and prognosis. Pathology. 1980; 12(3):439-47.

[15] Wagener C, Müller-Wallraf R, Nisson S, Gröner J, Breuer H. Localization and concentration of carcinoembryonic antigen (CEA) in gastrointestinal tumors: correlation with CEA levels in plasma. J Natl Cancer Inst. 1981; 67(3): 539-47.

[16] Nikseresht M, Seghatoleslam A, Monabati A, Talei A, Ghalati FB, Owji AA. Overexpression of the nove human gene, UBE2Q2, in breast cancer. Cancer Genet Cytogenet. 2010;197(2):101-6.

[17] Mrayam R. Study the effect of UBE2Q1over expression on the protein level of p53 and the expression of cyclin E,BCL2 and MDM2 genes in LS180 and SW480 colorectal cancer cell lines: Shiraz University of Medical Sience; 2015.

[18] Garraway LA, Lander ES. Lessons from the cancer genome. Cell. 2013; 153(1):17-37.

[19] Galluzzi L, Pietrocola F, Bravo-San Pedro JM, Amaravadi RK, Baehrecke EH, Cecconi F, et al. Autophagy in malignant transformation and cancer progression. EMBO J. 2015; 34(7):856-80.

[20] Chang R, Wei L, Lu Y, Cui X, Lu C, Liu L, et al. Upregulated expression of ubiquitin-conjugating enzyme E2Q1 (UBE2Q1) is associated with enhanced cell proliferation and poor prognosis in human hapatocellular carcinoma. J Mol Histol. 2015; 46(1):45-56.

[21] Schmitz KJ, Ademi C, Bertram S, Schmid KW, Baba HA. Prognostic relevance of autophagy-related markers LC3, p62/sequestosome 1, Beclin-1 and ULK1 in colorectal cancer patients with respect to KRAS mutational status. World J Surg Oncol. 2016; 14(1):189.

[22] Wu S, Sun C, Tian D, Li Y, Gao X, He S, Li T. Expression and clinical significances of Beclin1, LC3 and mTOR in colorectal cancer. Int J Clin Exp Pathol. 2015; 8(4): 3882.

[23] Nicholson BD, Shinkins B, Pathiraja I, Roberts NW, James TJ, Mallett S, Perera R, Primrose JN, Mant D. Blood CEA levels for detecting recurrent colorectal cancer. Cochrane Database Syst Rev. 2015(12): CD011134.

[24] Palos G, Gilmore KR, Chapman P, Lewis-Patterson P, Bi W, Rodriguez MA. Low concordance with CEA tumor marker monitoring in colorectal cancer survivors. Cancer Res. 2016; 7(14): 3464. 